\title{
Preparation of Hollow Silica Nanoparticles Using Fluorinated Surfactant and Fluorocarbon Solvents
}

Tổng hợp vật liệu nano silica rỗng sử dụng chất hoạt động bề mặt và dung môi fluorocarbon

\author{
Thanh Khoa Phung ${ }^{1,2}, \mathrm{Ha}$ N. Giang ${ }^{3}$, Khanh B. Vu ${ }^{1,2^{*}}$ \\ ${ }^{1}$ School of Biotechnology, International University, Ho Chi Minh City, Vietnam \\ ${ }^{2}$ Vietnam National University, Ho Chi Minh City, Vietnam \\ ${ }^{3}$ Ho Chi Minh City University of Food Industry, Ho Chi Minh City, Viet Nam \\ *Email:vubkhanh@gmail.com
}

\begin{abstract}
In this study, we used a new class of fluorinated surfactant as a soft template for the preparation of the hollow silica nanoparticles. The size of the hollow silica nanoparticles was enlarged by incorporating a variety of swelling agents (perfluorodecalin, perfluorotributylamine, perfluorooctane, and perfluorooctyl bromide) into the cores of the micelles of the fluorinated surfactant. However, once we used the perfluorinated acids (perfluorooctadecanoic acid and perfluorodecanoic acid) as swelling agents, the structure of silica nanoparticles is solid without the formation of hollow voids. The TEM analysis combined with copper elemental mapping of the hollow silica loaded with copper hexadecafluorophthalocyanine indicated that the cores of the hollow silica nanoparticles are hydrophobic. The formation mechanism of the hollow silica nanoparticles is similar to that prepared by hydrocarbon surfactant/hydrocarbon, which was supported by the zeta potential measurements. The prepared hollow silica nanoparticles had the type IV isotherm with the H3 hysteresis loop.
\end{abstract}

Keywords: Hollow silica, fluorinated surfactant, fluorocarbon, micelle, swelling agent

Tóm tắt

Trong nghiên cứu này, chúng tôi sử dụng chất hoạt động bề mặt fluorocarbon làm khuôn mềm để tổng hợp các hạt nano silica rỗng. Kích thước của các hạt nano silica rỗng được tăng lên bằng cách thêm vào các chất gây trương nở như perfluorodecalin, perfluorotributylamine, perfluorooctane và perfluorooctyl bromide vào lõi của mixen. Tuy nhiên, khi chúng tôi sử dụng axit perfluorooctadecanoic và axit perfluorodecanoic làm tác nhân trương nở thì cấu trúc của hạt nano silica là rắn mà không hình thành các lỗ rỗng. Phân tích TEM kết hợp nguyên tố đồng của silica rỗng chứa đồng hexadecafluorophthalocyanine chỉ ra rằng lõi của các hạt nano silica rỗng có tính ky nước. Cơ chế hình thành của các hạt nano silica rỗng tương tự như được điều chế bằng chất hoạt động bề mặt và dung môi hydrocarbon. Các hạt nano silica rỗng có đường hấp phụ đẳng nhiệt loại IV với vòng trễ loại H3.

Từ khóa: Silica rỗng, chất hoạt động bề mặt fluorocarbon, mixen, tác nhân gây trương nở

\section{Introduction}

Hollow nanoparticles have attracted much attention because they exhibit many potential applications in delivery systems, catalysts, sensors, storage materials, photonic materials, and nanoreactors [1]. Hollow nanoparticles have been typically synthesized by soft templates and hard templates. The soft templates can be from the assembly of small surfactant molecules or polymeric chains with or without swelling agents [2-5]. The hard templates are usually polymeric nanoparticles or metallic oxides. The most typical hard template is polystyrene with positive charge on its surface [6,7] or polystyrene functionalized with siloxane groups [8]. Other hard templates are carbon [9], silica [10], and calcium carbonate particles $[11,12]$. The intermediate approach between soft and hard

ISSN: 2734-9381

https://doi.org/10.51316/jst.149.etsd.2021.31.2.21

Received: February 11, 2020; accepted: July 22, 2020 templates is the nanoprecipitation of polymer to form nanoparticle templates for growing the hollow shells [13-15]. This nanoprecipitation is easy to be performed and a variety of polymers can be selected.

The templates need to be removed from the composite particles after the synthesis to create the hollow structure. The removal of templates can be performed by solvent extraction or calcination at temperature that is suitable for the pyrolysis of templates. The size of hollow nanoparticles can be modified by using swelling agents in soft templates or by varying the diameter of hard template nanoparticles.

Usually, the polymeric hard template provides bigger size of hollow nanoparticles than the soft templates; however, the hard template approach gives better particle size distribution than the soft templates. The poor size distribution of hollow nanoparticles using the soft templates originates from the flexible dynamics of micelles of small molecules 
or polymeric chains in solution. The nature of templates (soft or hard) in the above-mentioned studies originates from hydrocarbon molecules (surfactants, polymeric beads, and swelling agents).

In this study, we aim at using a fluorinated surfactant (FS) as a soft template and a variety of fluorocarbon solvents (perfluorodecalin, perfluorotributylamine, perfluorooctane, perfluorooctyl bromide, perfluorooctadecanoic acid, and perfluorodecanoic acid) as swelling agents for the synthesis of the hollow silica nanoparticles. We also incorporated copper hexadecafluorophthalocyanine into the cores of the hollow silica nanoparticles to support the fact that the cores of the hollow silica nanoparticles are hydrophobic, which is compatible with copper hexadecafluorophthalocyanine.

\section{Experimental}

\subsection{Synthesis of materials}

Synthesis of cationic fluorinated surfactant: The details of synthesis and chemical analysis for this surfactant can be found in the previous work (cf. Supporting Information) [16].

Synthesis of the hollow silica using fluorocarbon surfactant (FS) and swelling agents: fluorinated surfactant $(30 \mathrm{mg})$ was dissolved in a mixture of deionized water $(15 \mathrm{~mL})$ and ethanol $(2 \mathrm{~mL})$ to have a clear solution. The swelling agent (10 $\mu \mathrm{L}$ of perfluorodecalin, perfluorotributylamine, perfluorooctane, perfluorooctyl bromide, perfluorooctadecanoic acid, or perfluorodecanoic acid) was added to $1 \mathrm{~mL}$ of the prepared surfactant solution, and then sonication for $10 \mathrm{~min}$ was applied to produce the oil-in-water emulsion. The emulsion was cooled down to $25{ }^{\circ} \mathrm{C}$ before sodium hydroxide $0.2 \mathrm{M}(10 \mu L)$ and tetraethyl orthosilicate $(50 \mu \mathrm{L}$, $17 \% \mathrm{v} / \mathrm{v}$ in methanol) were added to this emulsion. The hydrolysis of tetraethyl orthosilicate to silica was performed at $25{ }^{\circ} \mathrm{C}$ for $24 \mathrm{~h}$ with stirring to get the solid precipitate. Hollow silica was retrieved by the centrifugation of the product at $6,411 \mathrm{~g}$ for $40 \mathrm{~min}$ and then re-dispersed in ethanol. This purification procedure was repeated three times to get the assynthesized hollow silica nanoparticles.

Synthesis of the hollow silica loaded with copper hexadecafluorophthalocyanine $\left(\mathrm{CuF}_{16} \mathrm{Pc}\right)$ : the mixture containing $60 \mathrm{~mL}$ of the prepared solution of the surfactant $(120 \mathrm{mg}$ FS in a mixture of $60 \mathrm{~mL}$ water and $8 \mathrm{~mL}$ ethanol), $0.6 \mathrm{~mL}$ of perfluorooctyl bromide, and $20.9 \mathrm{mg}$ of $\mathrm{CuF}_{16} \mathrm{Pc}$ was sonicated for $20 \mathrm{~min}$ to form a clear emulsion of oil-in-water. After that, $\mathrm{NaOH} \quad 0.2 \quad \mathrm{M}(600 \mu \mathrm{L})$ and tetraethyl orthosilicate $(3 \mathrm{~mL}, 20 \% \mathrm{v} / \mathrm{v}$ in methanol) were added to the emulsion. The reaction mixture was stirred at $25{ }^{\circ} \mathrm{C}$ for $24 \mathrm{~h}$. After that, the mixture was centrifuged at $6,411 \mathrm{~g}$ for $40 \mathrm{~min}$ and then re-dispersed in ethanol. This purification procedure was repeated three times to get the as-synthesized nanoparticles. The as-synthesized sample was calcined at $500{ }^{\circ} \mathrm{C}$ for $6 \mathrm{~h}$.

\subsection{Characterizations of materials}

The size of the hollow silica nanoparticles was characterized by transmission electron microscopy (TEM, FEI Titan G2 80-300) and dynamic light scattering (Zetasizer Nano ZS). The elemental mapping was performed by TEM (FEI Titan G2 80-300). The morphology was characterized by scanning electron microscopy (SEM, FEI Quanta 200 FEG), and the textural properties were measured by the nitrogen-sorption technique (Micromeritics ASAP 2420). Zeta potential was measured by the Zetasizer Nano ZS.

\section{Results and discussion}

We enlarged the size of micelles of fluorinated surfactant by incorporating several swelling agents into the core of the FS micelles. The swelling agents we selected were perfluorodecalin, perfluorotributylamine, perfluorooctane, perfluorooctyl bromide, perfluorooctadecanoic acid, and perfluorodecanoic acid. The proposed swelling mechanism is shown in Fig. 1 and is supported by the zeta potential measurement of the micellar solution before and after being coated by silica species.

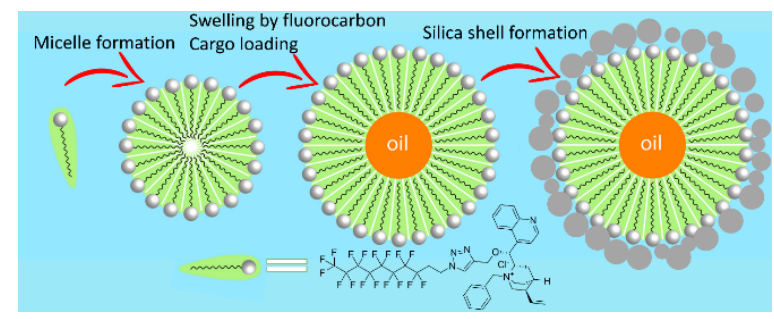

Fig. 1. The proposed mechanism of the formation of the hollow silica nanoparticles using fluorocarbon chemistry.

The fluorinated surfactant was chosen because the fluorinated tails assemble to form the cores of the micelles in the aqueous solution. The fluorinated cores of the micelles are compatible with fluorocarbon solvent, and therefore fluorocarbon swelling agent can diffuse into the cores of the micelles, or the micelles re-distribute to be adsorbed on the surfaces of fluorocarbon droplets. The zeta potential of the fluorinated micelles in aqueous solution is positive $(+46.0 \mathrm{mV})$ at $\mathrm{pH}=12.0$ because the fluorinated surfactant heads are positively charged, which are compatible with the aqueous phase. The interaction between the positively charged micelles of fluorinated surfactant and negatively charged silica species is direct co-condensation following the electrostatic interaction mechanism. After the fluorinated micelles (containing fluorocarbon) being coated with silica species, the zeta potential of the silica nanoparticles shows a 
negative value $(-21.5 \mathrm{mV})$, indicating that micelles were covered by silica species that have a negative value of zeta potential in an alkaline solution.

The as-synthesized silica samples were characterized by TEM, and the results are shown in Fig. 2.

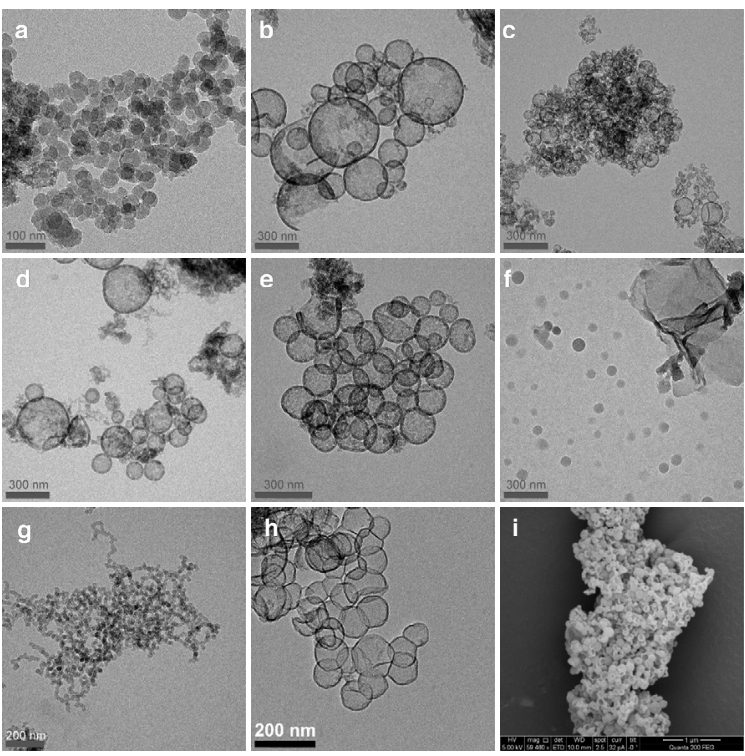

Fig. 2. TEM images of the as-synthesized silica samples using (a) FS surfactant, (b) FS/perfluorodecalin, (c) FS/perfluorotributylamine, (d) FS/perfluorooctane, (e) FS/perfluorooctyl bromide, (f) FS/perfluorooctadecanoic acid, (g) FS/perfluorodecanoic acid, and (h) FS/perfluorooctyl bromide with $\mathrm{CuF}_{16}$ PC. (i) SEM image of the hollow silica nanoparticles using FS/perfluorooctyl bromide with $\mathrm{CuF}_{16} \mathrm{PC}$.

The TEM image of silica sample without a swelling agent is presented in Fig. 2a. This sample exhibits hollow nanoparticles with a mean diameter of $44( \pm 5) \mathrm{nm}$ and a void diameter of $5 \mathrm{~nm}$. The size of the hollow silica nanoparticles pronouncedly changes as the swelling agent (perfluorodecalin, perfluorotributylamine, perfluorooctane, or perfluorooctyl bromide) was used, as seen through Figs. 2b, c, d, and e.

The sizes of the hollow silica nanoparticles and of the voids with swelling agents become much bigger as compared with the hollow silica nanoparticles prepared by the FS only. Particularly, perfluorodecalin (PFD) swelling agent gives the mean diameter of the hollow silica nanoparticles of $220 \mathrm{~nm}$ (standard deviation $=107 \mathrm{~nm}, \min =92 \mathrm{~nm}$, $\max =483 \mathrm{~nm}$ ). Perfluorotributylamine (PFTA) swelling agent gives the mean diameter of the hollow silica nanoparticles of $92 \mathrm{~nm}$ (standard deviation $=30 \mathrm{~nm}, \min =39 \mathrm{~nm}, \max =142 \mathrm{~nm})$. Perfluorooctane (PFO) swelling agent gives the mean diameter of the hollow silica nanoparticles of $162 \mathrm{~nm}$ (standard deviation $=73 \mathrm{~nm}, \min =93 \mathrm{~nm}$, $\max =364 \mathrm{~nm})$. Perfluorooctyl bromide (PFOB) swelling agent gives the mean diameter of the hollow silica nanoparticles of $155 \mathrm{~nm}$ (standard deviation $=40 \mathrm{~nm}, \min =77 \mathrm{~nm}, \max =241 \mathrm{~nm})$. The thickness of the shell of those hollow silica nanoparticles with the presence of the swelling agent is $16 \mathrm{~nm}$.

On the contrary, perfluorooctadecanoic acid (PFOA) and perfluorodecanoic acid (PFDA) do not play as the swelling agents, as seen in Figs. $2 \mathrm{f}$ and $\mathrm{g}$, because the hollow structure of the silica nanoparticles was not observed in those cases. Perfluorooctadecanoic acid produces the solid silica nanoparticles with the mean diameter of $67 \mathrm{~nm}$ (standard deviation $=12 \mathrm{~nm}, \min =47 \mathrm{~nm}$, $\max =95 \mathrm{~nm}$ ), and perfluorodecanoic acid produces the solid silica nanoparticles with the mean diameter of $23 \mathrm{~nm}$ (standard deviation $=3 \mathrm{~nm}, \min =18 \mathrm{~nm}$, $\max =29 \mathrm{~nm}$ ). For the ease of comparison, the outer diameter of the hollow silica obtained with a variety of swelling agents was summarized in Table 1.

Table 1. The outer diameter of the hollow silica obtained with a variety of swelling agents.

\begin{tabular}{|l|c|c|c|c|c|c|}
\hline & PFD & PFTA & PFO & PFOB & PFOA & PFDA \\
\hline mean & 220 & 92 & 162 & 155 & 67 & 23 \\
\hline SD & 107 & 30 & 73 & 40 & 12 & 3 \\
\hline $\min$ & 92 & 39 & 93 & 77 & 47 & 18 \\
\hline $\max$ & 483 & 142 & 364 & 241 & 95 & 29 \\
\hline
\end{tabular}

The hollow structure of the silica nanoparticles with swelling agents suggests that the swelling agent has formed the nanoemulsion stabilized by the FS surfactant in the aqueous medium. The FS surfactant adsorbed on the surface of the nanoemulsion through a hydrophobic - hydrophobic interaction between the fluorinated tail of the FS surfactant and the surface of nanoemulsion. Consequently, the FS surfactant and swelling agent formed a positively charged surface where the negatively charged silica species interacted with the positively charged heads of the FS surfactant and formed a silica layer on that surface, as postulated in the possible mechanism in Fig. 1. On the contrary, the acidic swelling agent consisting of negatively charged carboxylates covered the surface of the FS micelles through the electrostatic interaction. Therefore, solid silica nanoparticles were formed outside the micelles because the negatively charged silica species and negatively charged heads of the fluorinated acid repulse to each other.

The hollow structure of silica nanoparticles synthesized by the FS and perfluorooctyl bromide loaded with $\mathrm{CuF}_{16} \mathrm{PC}$ is presented in Figs. $2 \mathrm{~h}$ and $\mathrm{i}$. We selected perfluorooctyl bromide swelling agent for this experiment because $\mathrm{CuF}_{16} \mathrm{Pc}$ was easily dissolved in this solvent in comparison with the other swelling agents. To verify if $\mathrm{CuF}_{16} \mathrm{PC}$ has been 
successfully incorporated into the hollow silica nanoparticles, the copper elemental mapping was performed.

The TEM images, copper and silicon elemental mapping images of the hollow silica prepared by $\mathrm{FS} /$ perfluorooctyl bromide with the presence of $\mathrm{CuF}_{16} \mathrm{PC}$ are presented in Figs. $3 \mathrm{a}, \mathrm{b}$, c, and $\mathrm{d}$. The copper element can be clearly seen from the copper elemental mapping image (white areas from Fig. 3b).

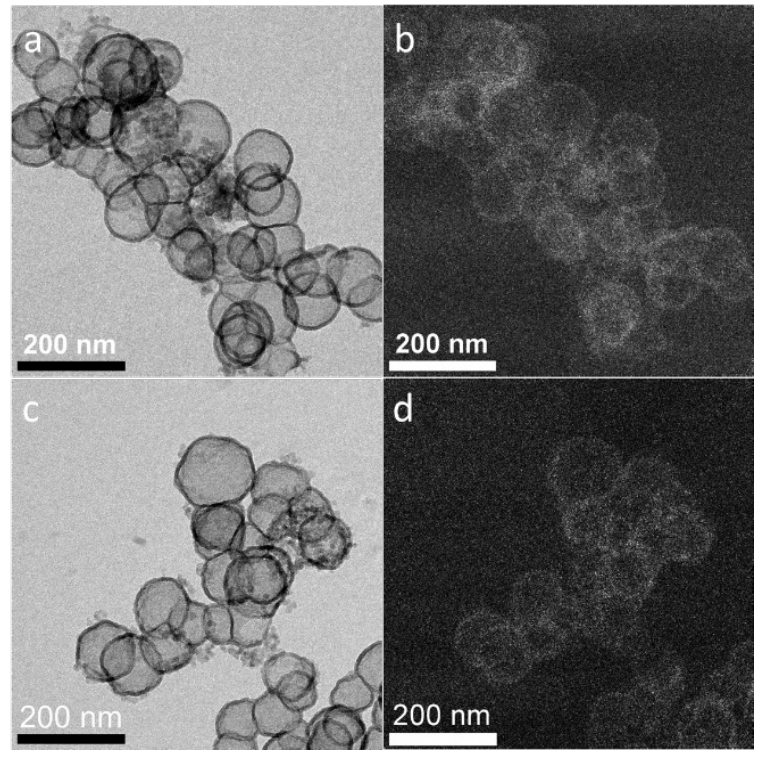

Fig. 3. TEM images of the as-synthesized silica samples using $(\mathrm{a}, \mathrm{c}) \mathrm{FS} /$ perfluorooctyl bromide with $\mathrm{CuF}_{16} \mathrm{PC}$, (b) its copper elemental mapping, and (d) silicon elemental mapping.

If we superpose the TEM image with its copper elemental mapping image, the white areas are well overlapped with those of the hollow silica nanoparticles. This observation indicates that $\mathrm{CuF}_{16} \mathrm{PC}$ has been integrated into the hollow silica nanoparticles and that nature of the cores of silica nanoparticles is hydrophobic. Similarly, the silicon element can be clearly seen from the silicon elemental mapping image (white areas from Fig. 3d), which indicates that the obtained particles are silica.

The textural properties of the hollow silica nanoparticles synthesized by FS and perfluorooctyl bromide loaded with $\mathrm{CuF}_{16} \mathrm{PC}$ before and after calcination at $500{ }^{\circ} \mathrm{C}$ for $6 \mathrm{~h}$ were measured by nitrogen sorption analysis. The isotherms and pore size distrutions are shown in Fig. 4. The isotherms of the hollow silica nanoparticles from both samples show the hysteresis loop that is characteristic for the type IV isotherm, which is associated with capillary condensation taking place in mesopores of the wall of hollow silica. The hysteresis loop of this material belongs to the type H3 loop, which does not exhibit any limiting adsorption at high $\mathrm{p} / \mathrm{p}_{0}$ This H3 loop is often observed with aggregates of plate-like nanoparticles giving rise to slit-shaped pores [17]. However, this type of $\mathrm{H} 3$ hysteresis loop has been also observed in hollow nanoparticles with mesoporous walls $[18,19]$. The BET surface areas of the as-synthesized and calcined samples are 288 and $915 \mathrm{~m}^{2} / \mathrm{g}$, respectively.

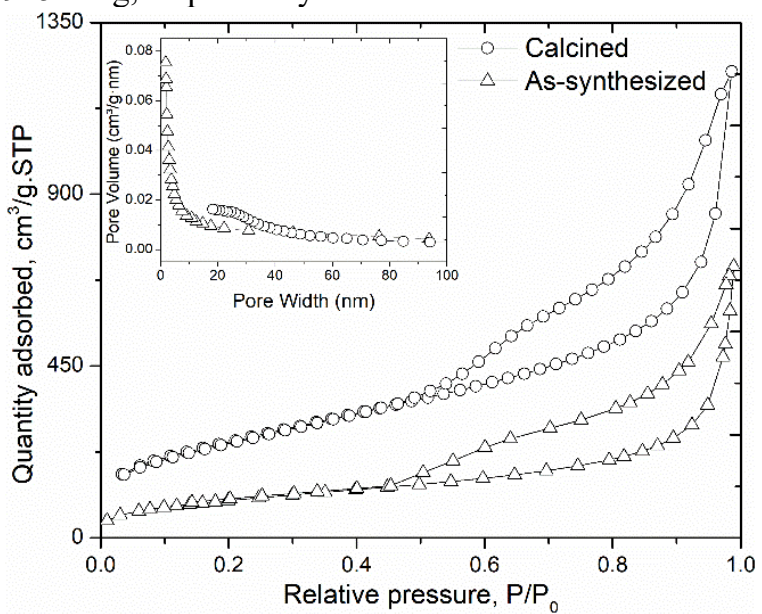

Fig. 4. Isotherm and pore size distribution (inset) of the silica sample prepared using FS/perfluorooctyl bromide with $\mathrm{CuF}_{16} \mathrm{PC}$. The as-synthesized powder sample was obtained by a freeze-drier. The calcined sample was obtained with the calcination of the as-synthesized sample at $500{ }^{\circ} \mathrm{C}$ for $6 \mathrm{~h}$.

In comparison with the as-synthesized sample, an increase in the surface area of the calcined sample indicates that the combustion of the organic compounds (surfactant, swelling agent, and $\mathrm{CuF}_{16} \mathrm{Pc}$ ) from the as-synthesized sample creates porosity, which leads to the enhancement of the surface area. The pore size distributions (the inset of Fig. 4) of the hollow silica from both as-synthesized and calcined samples do not show any clear peaks that represent for mean diameter of the pores. This observation implies that the microporosity (from silica shells) and macroporosity (from hollow voids) are likely predominant in these materials.

\section{Conclusion}

In conclusions, we have successfully used a fluorinated surfactant to prepare the hollow structure of the silica nanoparticles. Perfluorodecalin, perfluorotributylamine, perfluorooctane, and perfluorooctyl bromide were successfully used as swelling agents to enlarge the size of the hollow silica nanoparticles. However, fluorinated acids such as perfluorooctadecanoic acid and perfluorodecanoic acid did not work as swelling agents because the obtained structure of the silica nanoparticles is solid. The repulsion of the negatively charged carboxylate functionals in fluorinated acids with the negatively charged silica species in aqueous solution may be responsible for the formation of solid structure of silica nanoparticles. The positive zeta potential of the fluorinated surfactant and negative zeta potential of the micelles of fluorinated surfactant coated with silica species propose that the formation of hollow structure of the silica nanoparticles prepared by 
fluorinated surfactant/fluorocarbon (swelling agent) is similar to that prepared by hydrocarbon surfactant/hydrocarbon (swelling agent). The hydrophobic nature of the cores of the silica nanoparticles was verified by the TEM analysis combined with the copper elemental mapping. This hydrophobic nature of the cores indicates that they can be loaded with other cargos than $\mathrm{CuF}_{16} \mathrm{PC}$ for certain applications.

\section{Acknowledgments}

This research is funded by Vietnam National Foundation for Science and Technology Development (NAFOSTED) under grant number 104.05-2018.47.

\section{References}

[1] R. Dong, W. Liu, J. Hao, Soft Vesicles in the Synthesis of Hard Materials, Acc. Chem. Res., 45 (2012) 504-513.

https://doi.org/10.1021/ar200124g

[2] J. Yuan, X. Bai, M. Zhao, L. Zheng, C12mimBr Ionic Liquid/SDS Vesicle Formation and Use As Template for the Synthesis of Hollow Silica Spheres, Langmuir, 26 (2010) 11726-11731.

https://doi.org/10.1021/la101221z

[3] Y.-S. Lin, S.-H. Wu, C.-T. Tseng, Y. Hung, C. Chang, C.-Y. Mou, Synthesis of hollow silica nanospheres with a microemulsion as the template, Chemical Communications, (2009) 3542-3544. https://doi.org/10.1039/B902681A

[4] Y. Li, B.P. Bastakoti, M. Imura, J. Tang, A. Aldalbahi, N.L. Torad, Y. Yamauchi, Dual SoftTemplate System Based on Colloidal Chemistry for the Synthesis of Hollow Mesoporous Silica Nanoparticles, Chemistry - A European Journal, 21 (2015) 6375-6380. https://doi.org/10.1002/chem.201406137

[5] Y. Bao, T. Wang, Q. Kang, C. Shi, J. Ma, Micelletemplate synthesis of hollow silica spheres for improving water vapor permeability of waterborne polyurethane membrane, Scientific Reports, 7 (2017) 46638. https://www.nature.com/articles/srep46638

[6] J. Yang, J.U. Lind, W.C. Trogler, Synthesis of Hollow Silica and Titania Nanospheres, Chemistry of Materials, 20 (2008) 2875-2877. https://doi.org/10.1021/cm703264y

[7] L. Zhang, M. D'Acunzi, M. Kappl, G.K. Auernhammer, D. Vollmer, C.M. van Kats, A. van Blaaderen, Hollow Silica Spheres: Synthesis and Mechanical Properties, Langmuir, 25 (2009) 27112717. https://doi.org/10.1021/la803546r

[8] I. Tissot, J.P. Reymond, F. Lefebvre, E. BourgeatLami, SiOH-Functionalized Polystyrene Latexes. A Step toward the Synthesis of Hollow Silica Nanoparticles, Chemistry of Materials, 14 (2002) 1325-1331.

https://doi.org/10.1021/cm0112441

[9] H. Zhang, H. Xu, M. Wu, Y. Zhong, D. Wang, Z. Jiao, A soft-hard template approach towards hollow mesoporous silica nanoparticles with rough surfaces for controlled drug delivery and protein adsorption, Journal of Materials Chemistry B, 3 (2015) 64806489.

https://doi.org/10.1039/C5TB00634A

[10] Q. Meng, S. Xiang, K. Zhang, M. Wang, X. Bu, P. Xue, L. Liu, H. Sun, B. Yang, A facile two-step etching method to fabricate porous hollow silica particles, Journal of Colloid and Interface Science, 384 (2012) 22-28.

https://doi.org/10.1016/j.jcis.2012.06.043

[11] Y. Nakashima, C. Takai, H. Razavi-Khosroshahi, W. Suthabanditpong, M. Fuji, Synthesis of ultra-small hollow silica nanoparticles using the prepared amorphous calcium carbonate in one-pot process, Advanced Powder Technology, 29 (2018) 904-908. https://doi.org/10.1016/j.apt.2018.01.006

[12] J.-F. Chen, H.-M. Ding, J.-X. Wang, L. Shao, Preparation and characterization of porous hollow silica nanoparticles for drug delivery application, Biomaterials, 25 (2004) 723-727. https://doi.org/10.1016/S0142-9612(03)00566-0

[13] C. Takai-Yamashita, H. Imabeppu, M. Fuji, Synthesis of hollow silica nanoparticles using poly (acrylic acid)-3,3'-diaminodipropylamine template, Colloids and Surfaces A: Physicochemical and Engineering Aspects, 483 (2015) 81-86. https://doi.org/10.1016/j.colsurfa.2015.07.022

[14] C. Takai-Yamashita, M. Ando, M. Noritake, H.R. Khosroshahi, M. Fuji, Emulsion templating of poly (acrylic acid) by ammonium hydroxide/sodium hydroxide aqueous mixture for high-dispersed hollow silica nanoparticles, Advanced Powder Technology, 28 (2017) 398-405.

https://doi.org/10.1016/j.apt.2016.10.010

[15] Y. Wan, S.-H. Yu, Polyelectrolyte Controlled LargeScale Synthesis of Hollow Silica Spheres with Tunable Sizes and Wall Thicknesses, The Journal of Physical Chemistry C, 112 (2008) 3641-3647. https://doi.org/10.1021/jp710990b

[16] V.A. Solovyeva, K.B. Vu, Z. Merican, R. Sougrat, V.O. Rodionov, One-Pot Synthesis of Au@SiO2 Catalysts: A Click Chemistry Approach, ACS Combinatorial Science, 16 (2014) 513-517. https://doi.org/10.1021/co5000932

[17] K.S.W. Sing, D.H. Everett, R.A.W. Haul, L. Moscou, R.A. Pierotti, J. Rouquerol, T. Siemieniewska, Reporting Physisorption Data for Gas Solid Systems with Special Reference to the Determination of Surface-Area and Porosity (Recommendations 1984), Pure Appl. Chem., 57 (1985) 603-619. https://doi.org/10.1002/9783527610044.hetcat0065

[18] B. Tan, H.J. Lehmler, S.M. Vyas, B.L. Knutson, S.E. Rankin, Fluorinated-Surfactant-Templated Synthesis of Hollow Silica Particles with a Single Layer of Mesopores in Their Shells, Adv. Mater., 17 (2005) 2368-2371. https://doi.org/10.1002/adma.200500344

[19] L. Zhang, S.Z. Qiao, Y.G. Jin, Z.G. Chen, H.C. Gu, G.Q. Lu, Magnetic Hollow Spheres of Periodic Mesoporous Organosilica and $\mathrm{Fe} 3 \mathrm{O} 4$ Nanocrystals: Fabrication and Structure Control, Adv. Mater., 20 (2008) 805-809.

https://doi.org/10.1002/adma.200700900 